Plant Tissue Cult. \& Biotech. 30(2): 231-242, 2020 (December)

CBangladesh Assoc. for Plant Tissue Culture \& Biotechnology

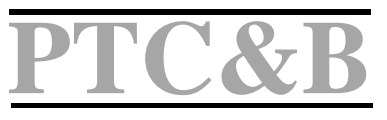

\title{
Genotypic Variability in Soybean [Glycine max (L.) Merrill] through Agrobacterium-Mediated Transformation
}

\author{
Shruti Shukla, Anita Rani*, Meeta Jain ${ }^{1}$ and Vineet Kumar \\ ICAR-Indian Institute of Soybean Research, Khandwa Road, Indore, M.P.-452001, India \\ Keywords: Agrobacterium-mediated transformation, Soybean, Genotypic variability, \\ GUSPlus
}

\begin{abstract}
Embryonic tip explants of 92 Indian soyabean and 7 advanced breeding lines derived from soaked mature seeds were inoculated and co-cultivated for 5-day with Agrobacterium strain EHA105 carrying the binary vector pCambia1305.1 containing a hygromycin and kanamycin resistance gene as plant and bacterial selectable markers, respectively. Transient expression of transgene was monitored by histochemical localization of $\beta$-glucouronidase (GUSPlus) reporter activity in transformed ET tissues. A high genetic variability for Agrobacterium-infection ranging from 3.8 to $100 \%$ was observed in the form of transient GUS expression. Five highly efficient genotypes, namely DS-228, JS 335, JS 72-44, KHSb2, and JS 72-280 with transient GUS expression of $100,98.1,96.5,96$ and $92 \%$, respectively were identified. In addition, various infectivity patterns in these genotypes were observed. Genotypes with very high transient GUS expression identified in this study may improve success rate of development of transgenic soybean.
\end{abstract}

\section{Introduction}

In 2018, the global area planted to soybean [Glycine max (L.) Merrill] was 123.5 million hectares, out of which $78 \%$ was biotech soybeans. The production area of biotech soybeans was 95.9 million hectares in 2018, comprising 69.3 million herbicide tolerance (HT) and 26.6 million hectares stacked insect resistance/HT.

The increment in income for farmers growing biotech soybean during 1996 to 2016 was US\$ 59.7 billion in which 2016 alone produced US\$ 6.9 billion (Brookes and Barfoot 2018).

\footnotetext{
*Author for correspondence: <anitavks@yahoo.co.in>. ${ }^{1}$ School of Biochemistry, Devi Ahilya
} Vishwavidyalaya, Khandwa Road, Indore, M.P.-452001.

$$
\text { DOI: https://doi.org/10.3329/ptcb.v30i2.50693 }
$$


Development of transgenic crop by transforming pre-existing genotypes with desired traits, such as high yield, insect, disease and herbicide resistance is the most important achievement of biotechnology.The most commonly applied methods for plant transformation are Agobacterium tumefaciens and microprojectile bombardment-mediated transformation. Over more than 20 years, both methods have been progressively improved and developed but A. tumefaciens-mediated transformation is preferred for transformation since it is less expensive easy to manipulate and produces lower transgene copy number with higher stable gene expression. Soybean transformation using A. tumefaciens was first time achieved by Hinchee et al. (1988). Agrobacteriummediated method is primarily used for soybean transformation, but the transformation efficiency is still relatively low (Chen et al. 2018) due to a number of factors affecting the efficiency of T-DNA delivery into the plant cell. These factors include plant and strain genotypes, explant types, explant damage, biotic shock, antibiotic stress and inoculation time (Mamidala and Nanna 2009, Wagiran et al. 2010).

Soybean transformation is genotype-dependent (Jia et al. 2015). Non availability of highly efficient genotype amenable to transformation is one of the major obstacles in the development of transgenic soybean as the susceptibility of soybean genotype to Agrobacterium infection plays a key role for the high level of genetic transformation efficiency. Genetic variability for susceptibility of soybean genotype to Agrobacterium infection exists in soybean gene pool as observed by Meurer et al. (1998). Therefore, screening soybean genotypes from the available germplasm resources suitable to Agrobacterium-mediated genetic transformation has become the focus for optimizing the soybean transformation system and improving transformation efficiency (Song et al. 2013) that offers a significant advancement for soybean breeding programs allowing the production of novel and genetically diverse plant materials. Genotypic dependency restricted the application of this method in the routine production of elite and commercially valuable soybean cultivars (Jia et al. 2015) as it needs time consuming process for transfer of transgene in commercial genotypes. If high transformation efficient genotypes are available in elite and commercially valuable cultivars, the success rate of transgenic soybean production would be improved considerably (Jia et al. 2015, Verma et al. 2014). Moreover, function of the most of soybean genes is not clear despite the availability of whole genome sequence. Availability of soybean genotype with high transformation efficiency will help in studying the function of gene response for various metabolic and physiological process of soybean plant.

The factors responsible for effect of genotypes on transformation efficiency are not clear. Shan et al. (2008) reported that some genetic factors are responsible for the susceptibility of genotypes to Agrobacterium infection in the transformation process. Genetics behind genotypic variability to transformation efficiency is defined by chromosomal and plasmid genomes of Agrobacterium strains which encode all the machinery necessary for attachment and T-DNA transfer. Explants of different plant genotypes produce inducer molecules varying in their inducing ability and cellular 
concentration which leads to different level of vir gene expression, thereby affecting their sensitivity to Agrobacterium-infection (Karami 2008). Bacterial ability and inability to synthesize and transfer sufficient T-DNA essential for a successful transformation is affected by high and low level of vir gene expression, respectively. Low susceptibility of genotype to Agrobacterium may be a result of the presence of inhibitors of the Agrobacterium sensory machinery. Zhang et al. (1999) noted that 2-hydroxy-4,7dimethoxybenzoxazin-3-one (MDIBOA) inhibits induction of vir gene expression by an unknown mechanism. MDIBOA is the major organic exudates present in maize seedling roots. Other than MDIBOA, IAA was also shown an inhibition mechanism to vir gene induction (Liu and Nester 2006).

A number of reports are available on differential susceptibility of various plant species to Agrobacterium infection (Porter 1991, Cheng et al. 2004). Genotypic variability have also been noted in various legumes (Atif at al. 2013, Hood et al. 1987, Owens and Cress 1984), maize (Ritchie et al. 1993), aspen (Beneddra et al. 1996), Pinus species (Bergmann and Stomp 1992), tomato (Van Roekel et al. 1993), Arabidopsis (Nam et al. 1997), and grape (Lowe and Krul 1991). Very few reports are available on genotypic variability to susceptibility of Agrobacterium infection in soybean (Song et al. 2013, Jia et al. 2015). The present study was undertaken to assess the genotypic variability of commercial cultivars and elite lines of soybean available in India.

\section{Materials and Methods}

A total of 92 diverse Indian soybean cultivars and 7 advanced breeding lines were tested for genotypic variability in the present study. Healthy and bold seeds were surfacesterilized by exposure to chlorine gas. The chlorine gas was prepared using a mixture of $5 \mathrm{ml} \mathrm{HCl}(39.6 \%)$ and $100 \mathrm{ml}$ sodium hypochlorite (4\%) and kept for 16 - $18 \mathrm{hrs}$ (Liu and Wei 2002).

Agrobacterium tumefacienss train EHA105 (Hood et al. 1993) containing binary vector pCambia1305.1 (CAMBIA, Australia; Fig. 1) was used for genetic transformation. pCambia1305.1(https:/wwww.markergene.com/pcambia-vectors) contained hygromycin phosphotran-ferase ( $h p t$ ) as the plant selectable marker, Kanamycin resistance gene as the bacterial selection marker and an intron containing GUSPlus gene from Staphylococcus as the reporter gene.

A. tumefaciens strain EHA105 was transformed with binary vector pCambia1305.1 by preparing heat-shock agro-competent cells of strain EHA105 following the protocol of Höfgen and Willmitzer (1988). The presence of pCambia1305.1 plasmid was confirmed in the antibiotic resistant bacteria colonies by colony PCR using $35 \mathrm{~S}$ promoter, nptII and GUS gene specific primers. Primer sequence and amplicon size is given in Table 1. 


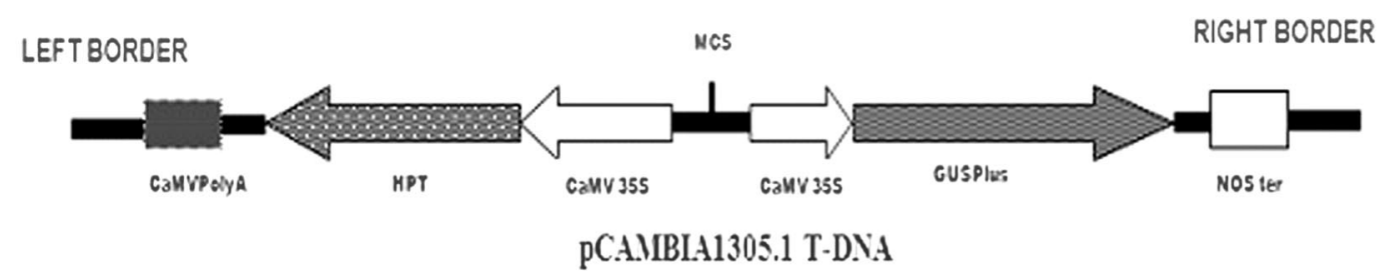

Fig. 1. Schematic diagram of T-DNA construct of binary vector pCAMBIA13051.1 used for genetic transformation of soybean, NOS ternpaline synthase terminator, GUSPlus coding region of the $\beta$-glucuronidase reporter gene, cauliflower mosaic virus polyadenylation signal.

Table 1. Sequence and product amplicon size of primers used for confirmation of transformed bacterial colonies.

\begin{tabular}{llcl}
\hline Primer & Sequence & Amplicon size (bp) & Reference \\
\hline 35S promoter & 'F' GCTCCTACAAATGCCATCA & 213 & Raharjo and \\
Specific primer & 'R'GATAGTGGGATTGTGCGTCA & & Surajiman 2017 \\
NPT I & 'F'TGCGCTGCGAATCGGGAGCG & 710 & Liu et al. 2004 \\
& 'R'GAGGCTATTCGGCTATGACT & & \\
GUS & 'F'CGACGGCCTGTGGGCATTCA & 900 & Rani et al. 2012 \\
& 'R TGGTCGTGCACCATCAGCAC & & \\
\hline
\end{tabular}

For infection Agrobacterium strain EHA105 containing pCambia1305.1 was grown on Luria agar (LA) plates (10 g/ casein enzymichydrolysate, $5 \mathrm{~g} /$ yeast extract and $10 \mathrm{~g} /$ $\mathrm{NaCl}, 1.5 \%$ agar; Himedia, India) containing $50 \mathrm{mg} / \mathrm{kanamycin}$ and rifampicin each at $28^{\circ} \mathrm{C}$ for 2 days. Single colony of Agrobacterium were obtained from the plate and inoculated into $50 \mathrm{ml}$ Luria broth (LB, liquid LA) containing $50 \mathrm{mg} /$ of both kanamycin and rifampicin (primary culture) for $6 \mathrm{hrs}$ at $28^{\circ} \mathrm{C}$ in $200 \mathrm{rpm}$. Subsequently, $500 \mu \mathrm{l}$ of the $50 \mathrm{ml}$ primary culture was mixed in a $200 \mathrm{ml} \mathrm{LB}$ culture, and grown overnight at $28^{\circ} \mathrm{C}$ in $200 \mathrm{rpm}$ using a shaker incubator. On the day of infection, bacterial pellet was obtained by centrifuging the overnight culture at $4000 \mathrm{rpm}$ for $10 \mathrm{~min}$ and re-suspended in infection medium containing 1/10 Gamborg's B5 medium (Gamborg et al. 1968) supplemented with $2.3 \mathrm{~g} / 2$-[N-morpholino] ethanesulfonic acid, $3 \%(30 \mathrm{~g} /)$ sucrose $(\mathrm{pH}$ 5.4), filter sterilized $1.67 \mathrm{mg} \wedge$ 6-N6-benzylaminopurine, $0.25 \mathrm{mg} \Lambda$ gibberellic acid and 50 $\mathrm{mg} /$ acetosyringone. Bacteria cell density was adjusted to 0.7 OD 600 using a nanodrop spectrophotometer (Denovix) before infection of explants and incubated at room temperature for $30 \mathrm{~min}$ before infection.

One day before infection the sterilized seeds were soaked in autoclaved distilled water overnight in the dark at $24^{\circ} \mathrm{C}$. ET explants were prepared by making a longitudinal cut along the hilum to separate the cotyledons, and the seed coat was removed. The ET 
was then excised from the junctions of the hypocotyls and primary leaves on ET were removed to expose the meristem (Liu et al. 2004).

After explants preparation ET explants were immersed in A. tumifecience EHA105 suspension (0.7 OD600) harboring the binary vector pCAMBIA1305.1 for $10 \mathrm{~min}$, at room temperature. After inoculation, 10 - 15 ETs (apical regions directed upwards) were placed in sterile Petri dish $(90 \mathrm{~mm})$ containing semi-solid co-cultivation medium (CCM), which is composed of infection medium additionally with $0.06 \%$ agar-agar (Himedia, India), filter sterilized cystein $(200 \mathrm{mg} /)$ and dithiothreitol $(154.2 \mathrm{mg} \Lambda)$ with a piece of Whatman filter paper and then incubated at $24^{\circ} \mathrm{C}$ in dark for 5 days.

After 5 days of co-cultivation, histochemical GUS assay was performed following the method of Jefferson et al. (1987). Transformed ET explants were incubated overnight at $37^{\circ} \mathrm{C}$ in a solution containing $200 \mathrm{mmol} \Lambda$ sodium phosphate buffer (pH 7.0), $500 \mathrm{mmol} \Lambda$ ethylenediaminetetraacetic acid, $0.5 \% \mathrm{v} / \mathrm{N}$ Triton, $20 \mathrm{mmol} / \mathrm{K}$-ferricyanide, $20 \mathrm{mmol} / \mathrm{K}$ ferrocyanide, $20 \mathrm{mmol} / \mathrm{5}$-bromo-4-chloro-3-indoxyl- $\beta$-D-glucuronide-cyclohexy-lammonium salt (X-Gluc) (Himedia, India) and $20 \% \mathrm{v} / \mathrm{N}$ methanol. After incubation, tissues were washed several times with $70 \%$ ethanol until the complete removal of chlorophyll. The tissues showing blue color after the removal of chlorophyll was scored and GUS stained transformed ETs were counted as GUS-positive transformants.

Transient GUS expression (\%) in 99 soybean cultivars was determined in three biological replications and each biological replication was measured in triplicate, the means and standard deviations were calculated. The data were statistically assessed using the one-way ANOVA. The comparison of the variation between means was performed via Fisher least significant difference (LSD) value through DMRT at a significance level of $\mathrm{p}<0.05$.

\section{Results and Discussion}

A total of 92 Indian soybean cultivars and 7 advanced breeding lines were transformed with the Agrobacterium strains EHA105 carrying pCAMBIA1305.1vector expressing the GUSPlus reporter gene to see the effect of genotypic variability on Agrobacteriummediated transformation efficiency. Transient GUS expression was observed in transformed ET tissues (Fig. 2) after 5 days in co-cultivation media.

The result showed that transformed ET explants of some soybean genotypes stained intensely with the substrate X-Gluc (Fig. 2a,b), while transformed ETs of some genotypes stained weakly (Fig. 2c,d). Transient GUS expression calculated by dividing number of stained ETs by total number of ETs infected by Agrobacterium in the experiment and is listed in Table 2. Soybean genotypes showed wide variations for transient GUS expression ranging from 3.8 to $100 \%$. Twenty genotypes showed $>70 \%$ transient GUS expression indicating high susceptibility to Agrobacterium infection, 48 genotypes showed moderate expression ranging from 30 to $70 \%$ and 31 genotypes were weakly susceptible showing <30\% Agrobacterium infection. Five genotypes, DS -228 (100\%), JS 335 (98.1\%), JS 
72 - 44 (96.5\%), KHSb 2 (96\%), and JS 72-280 (92\%) were highly sensitive to Agrobacterium infection. In contrast, five genotypes, namely Pusa 98 - 14 (3.8\%), Davis (7\%), MACS-58 (7), PK 1092 (7.2\%), and Palam soya (8.3\%) showed the lowest transformation efficiency.

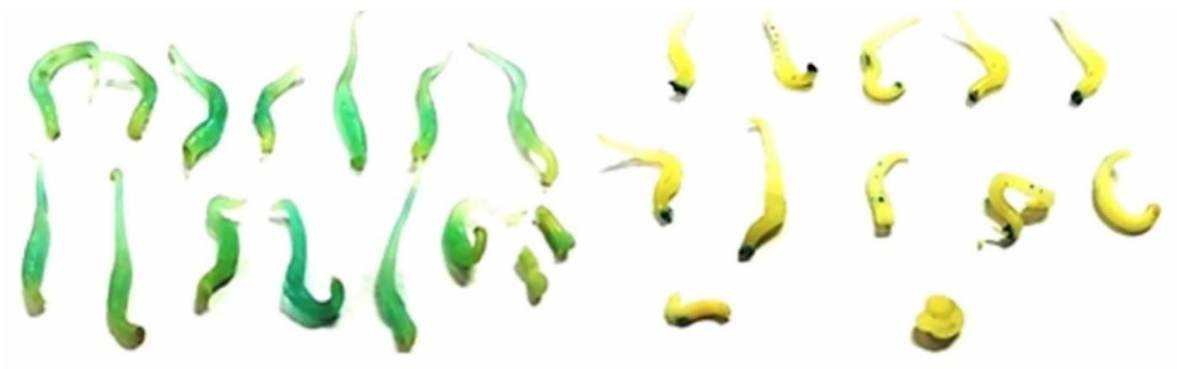

(a)

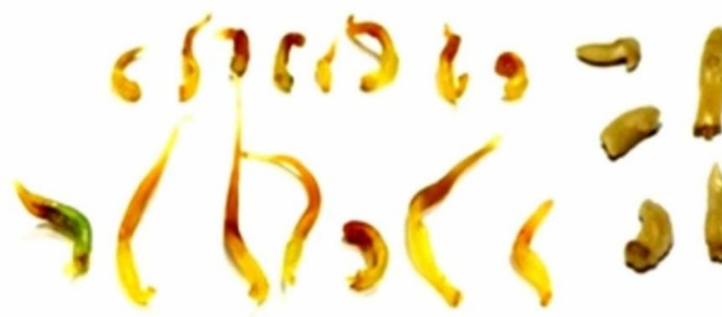

(c)

(b)

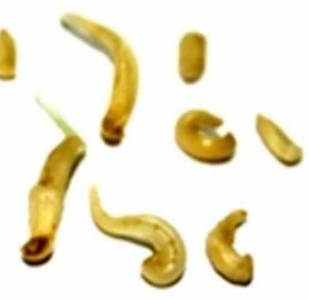

(d)

Fig. 2. Histochemical GUS staining of transformed ET explants infected with Agrobacterium EHA105 containing pCambia1305.1 vector. (a) Highly susceptible genotype KHSb 2 showing GUS expression covering all parts of explant, (b) highly susceptible genotype JS 72-280 showing GUS expression at shoot meristem only, (c) weakly susceptible genotypes ACS-58 and (d) showing GUS expression in a few explants with very weak blue color intensity.

Present study also proved that Agrobacterium-transformation efficiency is highly affected by plant genotypes. The effect of cultivars on transient GUS expression was found to be highly significant. The transient GUS expression of DS228 was found be the highest $(100 \%)$ and transient GUS expression of JS $335(98.1 \%)$ was not significantly lower than DS 228 but significantly higher than all the other varieties examined under the study (Table 2).

It was also observed genetic relatedness among genotypes showed high susceptibility to Agrobacterium infection. All the genotypes, which have JS 335 (98.1\%) as one of its parent showed high transient GUS expression $(>70 \%)$, suggesting the role of genetic factors. DS - $228(100 \%)$ and MAUS - $81(73.1 \%)$ showing high transient GUS expression are evolved from the cross JS $335 \times$ DS 181 and KB $-74 \times$ JS 335, respectively. Similarly, NRCSL1 and NRCSL2, advanced breeding lines both developed from cross between JS335 and SL525 showed 86 and 72\% transient GUS expression, respectively. Though Co 3 developed from UGM $69 \times$ JS 335 did not show high transient GUS expression (52\%). 
Table 2. Evaluation of genotypic variability of Indian soybean for Agrobacterium-mediated transformation efficiency.

\begin{tabular}{|c|c|c|c|c|c|}
\hline $\begin{array}{l}\text { Genotype } \\
\text { number }\end{array}$ & $\begin{array}{l}\text { Soybean } \\
\text { genotype }\end{array}$ & $\begin{array}{l}\text { Transient GUS } \\
\text { expression (\%) }\end{array}$ & $\begin{array}{l}\text { Genotype } \\
\text { number }\end{array}$ & $\begin{array}{l}\text { Soybean } \\
\text { genotype }\end{array}$ & $\begin{array}{l}\text { Transient GUS } \\
\text { expression (\%) }\end{array}$ \\
\hline 1 & DS-228 & $100 \pm 0^{\mathrm{a}}$ & 51 & JS93-05 & $42.3 \pm 0.7 \mathrm{uv}$ \\
\hline 2 & JS 335 & $98.1 \pm 1.0^{\mathrm{ab}}$ & 52 & SL-96 & $42.3 \pm 1.1^{\mathrm{uv}}$ \\
\hline 3 & JS 72-44 & $96.5 \pm 1.6^{\mathrm{b}}$ & 53 & Co 2 & $40.5 \pm 0.6^{\mathrm{vw}}$ \\
\hline 4 & KHSb2 & $96.0 \pm 1.0^{\mathrm{b}}$ & 54 & Bragg & $40.4 \pm 1.4^{\mathrm{vw}}$ \\
\hline 5 & JS 72-280 & $92 \pm 3.0^{c}$ & 55 & MAUS-57 & $40.2 \pm 1.2^{\mathrm{vw}}$ \\
\hline 6 & MAUS61-2 & $89.4 \pm 0.6^{c}$ & 56 & MACS-57 & $40.1 \pm 1.0^{\mathrm{vw}}$ \\
\hline 7 & NRCSL1 & $86 \pm 1.7^{\mathrm{d}}$ & 57 & PK 1042 & $39.6 \pm 1.4^{\mathrm{vw}}$ \\
\hline 8 & JS95-60 & $85.4 \pm 0.9^{\mathrm{d}}$ & 58 & Birsa Soya-1 & $38.3 \pm 2.2^{w x}$ \\
\hline 9 & PK 471 & $85.3 \pm 0.8^{\mathrm{d}}$ & 59 & MAUS-47 & $37.2+2.1^{w x y}$ \\
\hline 10 & PRS1 & $85.0 \pm 0.8^{\mathrm{d}}$ & 60 & JS 2034 & $35.5 \pm 1.8 \mathrm{xyz}$ \\
\hline 11 & PK 564 & $84.9 \pm 0.9 \mathrm{~d}$ & 61 & Monetta & $35.3 \pm 1.1 \mathrm{xyz}$ \\
\hline 12 & JS 76-205 & $83.8 \pm 1.1^{\mathrm{de}}$ & 62 & Indira Soya & $35.1 \pm 1.0^{x y z}$ \\
\hline 13 & MACS-124 & $83.7 \pm 0.5^{\mathrm{de}}$ & 63 & Pratap Soya 2 & $34.3 \pm 0.3 \mathrm{yzA}$ \\
\hline 14 & DSB1 & $82.6 \pm 2.5^{\mathrm{de}}$ & 64 & JS 79-81 & $33.6 \pm 1.3 \mathrm{zAB}$ \\
\hline 15 & Punjab-1 & $80.4+2.2^{\mathrm{e}}$ & 65 & Lee & $32.4 \pm 0.6^{\mathrm{zABC}}$ \\
\hline 16 & Pusa 37 & $75 \pm 1.6^{\mathrm{f}}$ & 66 & PS 1347 & $31.2 \pm 1.0^{\mathrm{ABCD}}$ \\
\hline 17 & NRC107 & $75 \pm 1.1^{\mathrm{f}}$ & 67 & Pusa 24 & $30.2 \pm 1.2^{\mathrm{BCDE}}$ \\
\hline 18 & Gujarati Soya-2 & $73.4 \pm 0.6 \mathrm{fg}$ & 68 & $\mathrm{~Kb}-79$ & $30 \pm 0.8 \mathrm{CDEF}$ \\
\hline 19 & MAUS-81 & $73.1 \pm 2.1^{\mathrm{fg}}$ & 69 & PS 1225 & $29.9 \pm 2.1 \mathrm{CDEFG}$ \\
\hline 20 & NRCSL2 & $72 \pm 2.8^{\mathrm{fg}}$ & 70 & Shilajeet & $28.2 \pm 1.0^{\mathrm{DEFGH}}$ \\
\hline 21 & JS 80-21 & $70 . \pm 1.6 \mathrm{gh}$ & 71 & PS 97-12 & $27.5 \pm 1.5^{\mathrm{EFGHI}}$ \\
\hline 22 & Pusa 22 & $68 . \pm 3.2^{\mathrm{hi}}$ & 72 & JS-2 & $27.3 \pm 4.5^{\mathrm{EFGHI}}$ \\
\hline 23 & JS97-52 & $65.5 \pm 0.8^{\mathrm{ij}}$ & 73 & Type49 & $26.6 \pm 1.8^{\mathrm{FGHI}}$ \\
\hline 24 & Pusa 16 & $65.3 \pm 2.3^{\mathrm{ijk}}$ & 74 & VLS47 & $26.4 \pm 2.1 \mathrm{GHIJ}$ \\
\hline 25 & MAUS-61 & $65.1 \pm 1.0 \mathrm{jk}$ & 75 & MAUS-158 & $26.3 \pm 1.1 \mathrm{HIJ}$ \\
\hline 26 & Kalitur & $63.5 \pm 0.5^{\mathrm{jkl}}$ & 76 & JS 2029 & $25.2 \pm 1.1^{\mathrm{HIJK}}$ \\
\hline 27 & NRC 37 & $62.3 \pm 1.2^{\mathrm{jklm}}$ & 77 & VLS2 & $24.3 \pm 4.0^{\mathrm{IIKL}}$ \\
\hline 28 & Improved Pelican & $61.9 \pm 2.4^{\mathrm{km}}$ & 78 & MAUS-32 & $23.0 \pm 1.0^{\mathrm{JKLM}}$ \\
\hline 29 & RKS-24 & $60.5 \pm 1.8 \mathrm{lmn}$ & 79 & PS 1024 & $23.0 \pm 1.0$ Ј КLМ \\
\hline 30 & PK 1029 & $60 \pm 1.1 \mathrm{mn}$ & 80 & Lsb1 & $22.1 \pm 0.8^{\mathrm{KLMN}}$ \\
\hline 31 & JS 75-46 & $58 \pm 4.0^{\text {no }}$ & 81 & NRC109 & $21.9 \pm 1.6^{\mathrm{KLMN}}$ \\
\hline 32 & SL295 & $55.9 \pm 1.3 \mathrm{op}$ & 82 & VLS1 & $21.8 \pm 1.7 \mathrm{KLMN}$ \\
\hline 33 & Alankar & $55.8 \pm 1.5^{\circ p}$ & 83 & Hara Soya & $21.1 \pm 1.4^{\mathrm{LMNO}}$ \\
\hline
\end{tabular}

(Contd.) 


\begin{tabular}{|c|c|c|c|c|c|}
\hline 34 & NRC 7 & $54.2 \pm 0.8 \mathrm{pq}$ & 84 & NRC138 & $19.9 \pm 2.7 \mathrm{MNOP}$ \\
\hline 35 & PK 472 & $53.8 \pm 1.4 \mathrm{pq}$ & 85 & PK 327 & $19.2 \pm 1.1^{\mathrm{NOP}}$ \\
\hline 36 & ADT-1 & $53.6 \pm 2.3 \mathrm{pq}$ & 86 & Pusa 40 & $18.6 \pm 0.7^{\mathrm{NOP}}$ \\
\hline 37 & PS 1241 & $52.2 \pm 0.8 \mathrm{qr}$ & 87 & JS 71-05 & $18.1 \pm 2.9 \mathrm{OP}$ \\
\hline 38 & Co 3 & $52 \pm 2.6^{\mathrm{qr}}$ & 88 & JS 90-41 & $17.2 \pm 0.8^{\mathrm{PQ}}$ \\
\hline 39 & NRC 2 & $50.1 \pm 1.1^{\mathrm{rs}}$ & 89 & SL688 & $14.3 \pm 2.0 \mathrm{QR}$ \\
\hline 40 & MAUS-2 & $50.1 \pm 1.1^{\mathrm{rs}}$ & 90 & NRC142 & $13.1 \pm 0.7^{\mathrm{RS}}$ \\
\hline 41 & Pusa 20 & $49.9 \pm 1.6^{\mathrm{rs}}$ & 91 & NRC127 & $11.5 \pm 2.4^{\mathrm{RST}}$ \\
\hline 42 & Tams-38 & $49.5 \pm 2.4^{\mathrm{rs}}$ & 92 & SL525 & $10.4 \pm 2.1^{\mathrm{STU}}$ \\
\hline 43 & Hardee & $49.4 \pm 3.1^{\mathrm{rs}}$ & 93 & Gujarati Soya-1 & $10.3 \pm 1.9 \mathrm{STU}$ \\
\hline 44 & PK 308 & $49.0 \pm 4.4^{\mathrm{rs}}$ & 94 & NRC 12 & $9.6 \pm 2.3^{\mathrm{TU}}$ \\
\hline 45 & MAUS-1 & $48.1 \pm 0.9^{\text {st }}$ & 95 & Palam soya & $8.3 \pm 1.2^{\mathrm{TU}}$ \\
\hline 46 & Ankur & $45.6 \pm 4.9^{\text {tu }}$ & 96 & PK 1092 & $7.2 \pm 0.6^{\mathrm{UV}}$ \\
\hline 47 & NRC 86 & $44.4 \pm 1.3^{\mathrm{u}}$ & 97 & Davis & $7 \pm 1.7 \mathrm{UV}$ \\
\hline 48 & Co 1 & $44.3 \pm 4.7 \mathrm{u}$ & 98 & MACS-58 & $7 \pm 0.3 \mathrm{UVz}$ \\
\hline 49 & PK 262 & $44.1 \pm 0.8 \mathrm{u}$ & 99 & Pusa 98-14 & $3.8 \pm 1.2^{\mathrm{V}}$ \\
\hline 50 & Shivalik & $42.7 \pm 4.6^{\mathrm{uv}}$ & & & \\
\hline \multicolumn{6}{|c|}{ LSD $(p=0.05) 3.09$} \\
\hline \multicolumn{6}{|c|}{ Main effect ${ }^{* * *}$} \\
\hline
\end{tabular}

"The data presented here are the average values of three replicates $\pm \mathrm{Sd}$. Values followed with dissimilar letters differ significantly at $\mathrm{p}=0.05$ in accordance with LSD and DMRT. The transient GUS expression was calculated as follows: Transient GUS expression (in $\%=$ The number of positive transformants/the total infected explants $\times$ 100. NRC numbers are 7 advanced breeding lines.

After infection and staining different Agrobacterium infectivity pattern in transformed explants was observed. Blue color was either on stem meristem (SM, Fig. 3a), coleoptiles region (CL, Fig. 3b), SM and CL both (Fig. 3c) or all part of the stained transformed ET explants (Fig. 3d). Blue loci developed covering all area of the stained transformed ET explants in genotypes, KHSb 2 and SL 295 (Figs 2a, 3d) and both SM and CL in JS 335; whereas, in JS72-280 and MAUS-2, it was observed only in SM and CL region, respectively (Fig. 3a, b). In contrast, low transformation efficient genotypes do not or less developed blue color after GUS staining (Fig. 2c, d). It is worth noting that DS-228 (100\%), JS 335 (98.1\%, Fig. 3c), JS72-44 (96.5\%), KHSb 2 (96\%, Fig. 2a), and JS 72-280 (92\%; Fig. 3a) showed high intense blue color with different infectivity patterns (SM, CL, both or all parts), especially at 5 days after co-cultivation, suggesting that Agrobacteriummediated transformation was more acceptable by high-efficient genotypes. Consequently, variations in color formation and intensity among the tested genotypes indicated that these differences were completely dependent on genotypes. 
Host plant genotype and Agrobacterium strain, both affect the level of Agrobacterium infection (Karami 2008). A number of studies have been reported genotypic variability in soybean (Song et al. 2013, Jia et al. 2015) and other crops like rice (Hoque et al. 2005), maize (Ritchie et al. 1993), various legumes (Hood et al. 1987; Owens and Cress 1984), aspen (Beneddra et al. 1996), Pinus species (Bergmann and Stomp 1992), tomato (van Roekel et al. 1993), Arabidopsis (Nam et al. 1997), and grape (Lowe and Krul 1991) for susceptibility to Agrobacterium infection. A variety of explants like cotyledonary node, half seed and ET have been used in development of transgenic soybean (Verma et al. 2011, Rani et al. 2012, Olhoft et al. 2003, Chen et al. 2018, Paz et al. 2006, Liu et al. 2004). ET was used for transient GUS expression in this study since it has many advantages over other explants. It is easier to obtain, shoot regeneration is direct, and is not prone to somaclonal variation and chromosomal abnormalities (Saeed et al. 1997). Moreover, the ET system has the highest regeneration frequency (Liu et al. 2004). This study was carried over to select genotypes of the most amenable to Agrobacterium infection as a first step to improve soybean transformation efficiency.

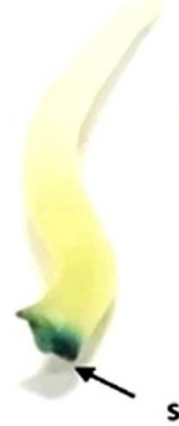

(a)

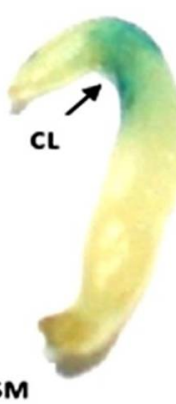

(b)

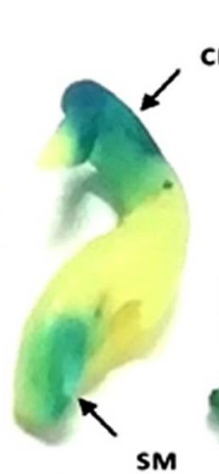

(c)

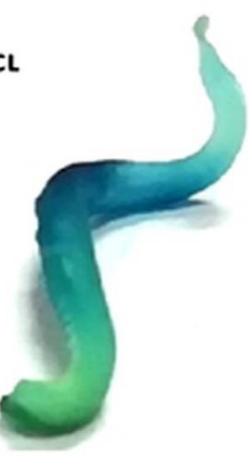

(d)

Fig. 3. Infectivity pattern of histocheical GUS expression in various regions of ET tissue observed after 5 days of co-cultivation when infected with pCambia 1305.1, (a) only stem meristem (SM) region stained in genotype JS72-280. (b) only coleoptiles (CL) region stained in MAUS-2, (c) both SM and CL regions stained in genotype JS 335, (d) complete tissue stained in SL 295.

This study mainly focused on cultivated variety and advanced breeding lines of India as identification of elite variety breeding line with high transformation efficiency will help in rapid development of transgenic soybean variety and there will be no need to introgress transgene from primary transgenic with poor agronomic background into high yielding variety. Significant genotype variability for susceptibility to Agrobacterium infection was observed in Indian soybean genotypes. This study screened 5 high-efficient genotypes DS-228, JS 335, JS 72-44, KHSb 2, and JS 72-280 with transient GUS expression of 100, 98.1, 96.5, 96 and 92\%, respectively and 5 weakly-susceptible genotypes Pusa9814, Davis, MACS-58, PK 1092 and Palam soya with low transient GUS expression of 3.8, 7, 7, 7.2 and $8.3 \%$, respectively (Table 2). JS335, a very high yielding Indian soybean 
variety,was many times used in transformation protocols (Verma et al. 2009, Rani et al. 2012) in earlier studies. This genotype showed high transient GUS expression (98.1\%) in this study, as well as four more genotypes, DS-228, JS72-280, JS 72-44, and KHSb2 also performed better (Table 2, Fig. 2). Five low-efficient genotypes, namely Davis, Palam soya, PK 1092, Pusa98-14, and MACS-58 displayed poor transient GUS expression. Jia et al. (2015) reported a strong defense response in the form of over expression of methyl jasmonate, polyphenol oxidase and peroxidase in weakly susceptible genotypes as compared to highly susceptible genotypes. Though these factors were not analyzed in this study, a mapping population has been developed from a cross of highly efficient genotype and genotype with very low efficiency to study the genetic factors responsible for the difference and map the genomic region regulating susceptibility to Agrobacterium infection. The highly efficient genotypes identified in present study can be used in development of transgenic soybean variety in India as all of the genotypes have been released for cultivation in India based on their yield performance in multi-location trial. Transgenics developed from these varieties can be directly released after legal clearance from the agencies authorized for release of transgenic crop varieties in India. These genotypes can also be used for studying function of genes with unknown roles to improve soybean performance under various biotic and abiotic stresses using virusinduced gene silencing (VIGS) or CRISPR-CAS9 techniques by silencing those genes.

\section{Acknowledgements}

Authors are thankful to Director, ICAR-IISR, Indore, M.P. for providing infrastructural facilities. This study was funded by ICAR-Indian Institute of Soybean Research, Indore.

\section{References}

Atif RM, Patat-Ochatt EM, Svabova L, Ondrej V, Klenoticova H, Jacas L, Griga M and Ochatt SJ (2013) Gene Transfer in Legumes. U. Lu“ttge et al. (eds.), Progress in Botany 74, DOI 10.1007 978-3-642-30967-0_2, Springer-Verlag Berlin Heidelberg

Beneddra T, Picard C and Nesme X (1996) Correlation between susceptibility to crown gall and sensitivity to cytokinin in aspen cultivars. Phytopathol. 86: 225-231.

Bergmann B and Stomp AM (1992) Effect of host plant genotype and growth rate on Agrobacterium tumefaciens-mediated gall formation in Pinus radiata. Phytopathol. 84: 1457-1462.

Brookes G and Barfoot P (2018) GM crops: Global socio-economic and environmental impacts 1996-2016. PG Economics Ltd, UK. pp. 1-204.

Chen L, Cai Y, Liu X, Yao W, Guo C, Sun S, Wu C, Jiang B, Han T and Hou W (2018) Improvement of Soybean Agrobacterium-mediated transformation efficiency by adding glutamine and asparagine into the culture media. Int. J. Mol. Sci. 19: 3039.

Cheng M, Lowe BA, Spencer TM, Ye XD and Armstrong CL (2004) Factors influencing Agrobacterium-mediated transformation of monocotyledonous species. In Vitro Cell Dev. Biol. Plant 40: 31-45. 
Gamborg OL, Miller RA and Ojima K (1968) Nutrient requirements of suspension cultures of soyabean root cells. Exp. Cell Res. 50: 151-158.

Hinchee MA, Connor-Ward DV, Newell CA, McDonell RE, Sato SJ, Gasser CS, Fishhoff DA, Re DB, Fraley RT and Horsch RB (1988) Production of transgenic soybean plants using Agrobacterium-mediated DNA transfer. Nat. Biotechnol. 6: 915-922.

Hofgen R and Willmitzer L (1988) Storage of competent cells for Agrobacterium transformation. Nucleic Acids Res. 16: 9877.

Hood EE, Fraley RT and Chilton MD (1987) Virulence of Agrobacterium tumefaciens strain A281 on legumes. Plant Physiol. 83: 529-534.

Hood EE, Gelvin SB, Melchers LS and Hoekema A (1993) New Agrobacterium helper plasmids for gene transfer to plants. Transgenic Res. 2: 208-218.

Hoque ME, Mansfield JW and Bennett MH (2005) Agrobacterium-mediated transformation of Indica rice genotypes: An assessment of factors affecting the transformation efficiency. Plant Cell Tiss. Org. 82: 45-55.

ISAAA (2018) Global Status of Commercialized Biotech/GM Crops in 2018. ISAAA Brief No. 54.ISAAA: Ithaca, New York. https://www.isaaa.org/resources/publications/biotech_crop_ annual_update. Accessed 10 June 2020.

Jefferson RA, Kavanagh TA and Bevan MW (1987) GUS fusions: $\beta$-glucronidase as a sensitive and versatile gene fusion marker in higher plants. EMBO J. 6: 3901-3907.

Jia Y, Yao X, Zhao M, Zhao Q, Du Y, Yu C and Xie F (2015) Comparison of soybean transformation efficiency and plant factors affecting transformation during the agrobacterium infection process. Int. J. Mol. Sci. 16: 18522-43.

Karami O (2008) Factors affecting Agrobacterium-mediated transformation of plants. Transgenic Plant J.2:127-37.

Liu P and Nester EW (2006) Indoleacetic acid, a product of transferred DNA, inhibits vir gene expression and growth of Agrobacterium tumefaciens C58. Proc. Natl. Acad. Sci. USA 103: 46584662.

Liu HK and Wei ZM (2002) A method for sterilizing mature seeds of soybean. Plant Physiol.Commun. 38: 260-272.

Liu HK, Yang C and Wei ZM (2004) Efficient Agrobacterium tumefaciens-mediated transformation of soybeans using an embryonic tip regeneration system. Planta 219: 1042-1049.

Lowe BA and Krul WR (1991) Physical, chemical, developmental, and genetic factors that modulate the Agrobacterium-Vitis interaction. Plant Physiol. 96: 121-129.

Mamidala P and Nanna RS (2009) Influence of antibiotics on regeneration efficiency in tomato. Plant Omics 2: 135.

Meurer CA, Dinkins RD and Collins GB (1998) Factors affecting soybean cotyledonary node transformation. Plant Cell Rep. 18: 180-186.

Nam J, Mysore KS, Zheng C, Knue MK, Matthysse AG and Gelvin SB (1999) Identification of TDNA tagged Arabidopsis mutants that are resistant to transformation by Agrobacterium. Mol. Gen. Genet. 261: 429-438.

Olhoft PM, Flagel LE, Donovan, CM and Somers DA (2003) Efficient soybean transformation using hygromycin B selection in the cotyledonary-node method. Planta 216: 723-735. 
Owens LD and Cress DE (1984) Genotypic variability of soybean response to Agrobacterium strains harboring the Ti or Ri plasmids. Plant Physiol. 77: 87-94.

Paz MM, Martinez JC, Kalvig AB, Fonger TM and Wang K (2006) Improved cotyledonary node method using an alternative explants derived from mature seed for efficient Agrobacteriummediated soybean transformation. Plant Cell Rep. 25: 206-213.

Porter JR (1991) Host range and implications of plant infection by Agrobacterium rhizogenes. Crit Rev Plant Sci. 10: 387-421.

Raharjo T and Surajiman S (2017) PCR primer specific CaMV 35S promoter to detect transgenic soybean in Indonesia commercial soybean and tempeh. Indones. J. Chem. 17: 415-421.

Rani A, Verma K and Saini R (2012) Recovery of fertile transgenic plants via Agrobacterium tumefaciens-mediated transformation in Indian soybean (Glycine max L. Merrill) cultivar. Indian J. Genet. Plant Breed. 72: 325-331.

Ritchie SW, Lui CN, Sellmer JC, Kononowicz, T, Hodges KH and Gelvin SB (1993) Agrobacterium tumefaciens-mediated expression of gus A in maize tissues. Transgenic Res. 2: 252-265.

Saeed NA, Zafar Y and Malik KA (1997) A simple procedure of Gossypium meristem shoot tip culture. Plant Cell, Tissue Organ Cul. 51: 201-207.

Shan Y, Xiaowei H and Xiurong W (2008) Assessment of factors affecting the transformation efficiency of soybean cotyledonary-node Agrobacterium-mediated transformation system. Mol. Plant. Breed.

Song ZY, Tian JL, Fu WZ, Li L, Lu LH, Zhou L, Shan ZH, Tang GX and Shou HX (2013) Screening Chinese soybean genotypes for Agrobacterium-mediated genetic transformation suitability. J. Zhejiang Univ-Sci. B14: 289-298.

Wagiran A, Ismail I, Zain CRCM and Abdullah R (2010) Agrobacterium tumefaciens mediated transformation of the isopentenyl transferase gene in japonica rice suspension cell culture. Aust. J. Crop Sci. 4: 421-429.

Van Roekel JS, Damm B, Melchers LS and Hoekema (1993) Factors influencing transformation frequency of tomato (Lycopersicon esculentum). Plant Cell Rep. 12: 644-647.

Verma K, Rani A and Saini R (2009) Direct organogenesis and plantlet regeneration from cotyledonary node of Indian soybean [Glycine max (L.) Merrill] cultivars. Soybean Res. 7: 8-15.

Verma K, Rani A and Saini R (2011) Efficient plant regeneration system from half seed explants of soybean [Glycine $\max (\mathrm{L}$.) Merrill]. Soybean Res. 8: 62.

Verma K, Saini R and Rani A (2014) Recent advances in the regeneration and genetic transformation of Soybean. J. Innov. Biol. 1: 015-026.

Zhang Z, Xing A, Staswick P and Clemente TE (1999) The use of glufosinate as a selective agent in Agrobacterium-mediated transformation of soybean. Plant Cell Tissue Organ Cult. 56: 37-46. 